\title{
KERNEL FUNCTIONS AND PARABOLIC LIMITS FOR THE HEAT EQUATION
}

\author{
BY JOHN T. KEMPER
}

Communicated by Wolfgang Wasow, May 4, 1970

Let $D \subset\{(x, t): t>0\}$ be a domain of the plane bounded by curves $x=\eta_{1}(t), x=\eta_{2}(t)$, and $t=0$, where $\eta_{1}(t)<\eta_{2}(t)$ for all $t$ and, for each $T \in(0, \infty), \eta_{i}(t)$ satisfies a Lipschitz condition with exponent $\frac{1}{2}$ on the interval $[0, T], i=1,2$. Let $(X, T) \in D$ and $\left(y_{0}, s_{0}\right) \in \partial D$ with $s_{0}<T$. A kernel function for the heat equation in $D$ at $\left(y_{0}, s_{0}\right)$ with respect to $(X, T)$ is a nonnegative solution of the heat equation in $D$, $K(x, t)$, which vanishes continuously on $\partial D-\left\{\left(y_{0}, s_{0}\right)\right\}$ and is normalized by the requirement that $K(X, T)=1$.

The notion of a kernel function has been studied in the case of harmonic functions in Lipschitz domains in $R^{n}$ by Hunt and Wheeden [3], whose results include a representation theorem for nonnegative harmonic functions and a proof of the almost everywhere (with respect to harmonic measure) existence of finite nontangential boundary values for harmonic functions having a one-sided bound in a Lipschitz domain. (See also [2].) The present note describes analogous results for the heat equation in regions of the plane.

Theorem. If $(X, T) \in D$ and $(y, s) \in \partial D$ with $s<T$, then there exists a unique kernel function for the heat equation in $D$ at $(y, s)$ with respect to $(X, T)$.

It is clear that, for $s<T$, a kernel function at $(y, s)$ with respect to $(X, T)$ is completely determined by its values in $D_{T}=\{(x, t) \in D: t<T\}$. Thus, it suffices to consider kernel functions at $(y, s)$ in the bounded region $D_{T}$. One is led to the following representation result.

Theorem. Let $\partial_{p} D_{T}$ denote the parabolic boundary of $D_{T}$, which is $\partial D_{T} \cap\{(x, t): t<T\}$, and for $(y, s) \in \partial_{p} D_{T}$, let $K(x, t, y, s)$ denote the value at $(x, t)$ of the kernel function at $(y, s)$ with respect to $(X, T)$. If $u(x, t)$ is any nonnegative temperature in $D_{T}$, then there exists a unique regular Borel measure $\mu$ on $\partial_{p} D_{T}$ such that

$$
u(x, t)=\int_{\partial_{p} D_{T}} K(x, t, y, s) d \mu(y, s) .
$$

AMS 1970 subject classifications. Primary 35K05; Secondary 35C15.

Key words and phrases. Heat equation, kernel function, parabolic limits, representation of positive temperatures. 
As was seen by Hunt and Wheeden and, previously, by Carleson [1], in the case of harmonic functions, such a representation leads to a statement of existence of certain limits at the boundary. In the harmonic case these are nontangential limits. For the domain $D$ considered here, a function $u(x, t)$ has parabolic limit $L$ at a point $(y, s) \in \partial D$ if $u(x, t)$ has limit $L$ at $(y, s)$ inside any parabolic cone contained in $D$ with vertex at $(y, s)$. (A parabolic cone is a set of the form either $\left\{(x, t): \alpha\left|t-s_{0}\right|^{1 / 2}<x-y_{0}<\beta\right\}$ or $\left\{(x, t): \alpha\left|x-y_{0}\right|^{2}\right.$ $\left.<t-s_{0}<\beta\right\}$, depending on whether the vertex $\left(y_{0}, s_{0}\right)$ is on the "side" or the "bottom" of $D$. Both $\alpha$ and $\beta$ are positive.)

We use the term "caloric measure" to denote the measure on $D$ which corresponds to harmonic measure in the case of Laplace's equation.

THEOREM. If $u(x, t)$ is a solution of the heat equation in $D_{T}$ such that $u(x, t)$ is nonnegative (or has a one-sided bound in $D_{T}$ ), then $u(x, t)$ has finite parabolic limits on $\partial_{p} D_{T}$ except for a set of zero caloric measure.

A similar theorem can be proven, assuming only that $u(x, t)$ has a one-sided bound in some parabolic cone at each point of $\partial_{p} D_{T}$. Furthermore, all of these theorems can be extended to domains $D$ of the form $D=\{(x, t): t>0, x>\eta(t)\}$, where $\eta(t)$ again satisfies a Lipschitz condition with exponent $\frac{1}{2}$ on intervals $[0, T]$ with $T<\infty$. Proofs of all the results mentioned here can be found in [4].

\section{REFERENCES}

1. L. Carleson, On the existence of boundary values for harmonic functions of several variables, Ark. Mat. 4 (1962), 393-399. MR 28 \#2232.

2. R. A. Hunt and R. L. Wheeden, On the boundary values of harmonic functions, Trans. Amer. Math. Soc. 132 (1968), 307-322. MR 37 \#1634

3. - Positive harmonic functions on Lipschitz domains, Trans. Amer. Math. Soc. 147 (1970), 507-527.

4. J. T. Kemper, Kernel functions and parabolic limits for the heat equation, Thesis, Rice University, Houston, Texas, 1970.

Rice University, Houston, Texas 77001 